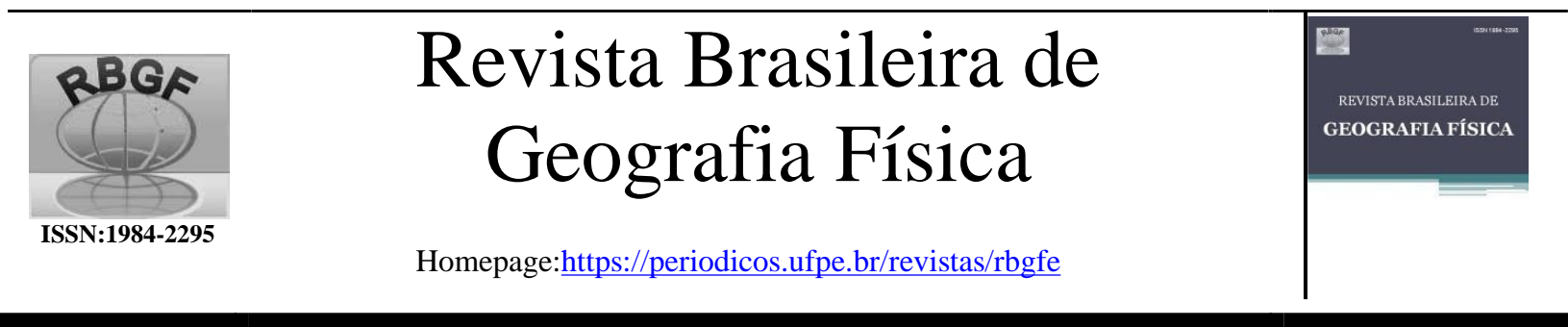

\title{
Caracterização Morfométrica e Uso e Ocupação do Solo em Bacia Hidrográfica do Semiárido Pernambucano
}

\author{
Antonio Genessis Bezerra dos Santos ${ }^{1}$, Alan Cezar Bezerra ${ }^{2}$, Antônio Henrique Cardoso do Nascimento ${ }^{3}$, Luciana \\ Sandra Bastos de Souza ${ }^{4}$, Jhonn Lennon Bezerra da Silva ${ }^{5}$, Geber Barbosa de Albuquerque Moura ${ }^{6}$ \\ 1 Graduando em Agronomia, Universidade Federal Rural de Pernambuco - Unidade Acadêmica de Serra Talhada, E-mail: \\ tonny.genessis123@hotmail.com \\ ${ }^{2}$ Professor Assistente, Universidade Federal Rural de Pernambuco - Unidade Acadêmica de Serra Talhada, E-mail: alan.bezerra@ $@$ ufrpe.br ${ }^{3}$ Professor \\ Adjunto, Universidade Federal Rural de Pernambuco - Unidade Acadêmica de Serra Talhada, E-mail: tonyagronomia@gmail.com ${ }^{4}$ Professora \\ Adjunto, Universidade Federal Rural de Pernambuco - Unidade Acadêmica de Serra Talhada, E-mail: sanddrabastos@yahoo.com.br ${ }^{5}$ Doutorando \\ em Engenharia Agrícola, Universidade Federal Rural de Pernambuco - Pós-Graduação em Engenharia Agrícola, E-mail: jhonlennoigt@hotmail.com \\ 6 Professor Associado, Universidade Federal Rural de Pernambuco - Departamento de Agronomia, E-mail: geber.moura@ufrpe.br
}

Artigo recebido em 07/04/2020 e aceito em 07/06/2020

\section{R E S U M O}

Este trabalho investigou a caracterização de uma bacia hidrográfica do Semiárido de Pernambuco e os efeitos da estiagem nas condições de uso e ocupação do solo por meio da adoção de geotecnologias aplicadas à gestão dos recursos hídricos. Para tanto, foram obtidas as imagens de altimetria pela Embrapa e realizada análise para caracterização morfométrica com o software QGIS e a extensão Terrain Analysis Using Digital Elevation Models TauDEM. Além disso, se utilizaram as imagens de uso e ocupação do solo da plataforma do MAPBIOMAS BRASIL referente aos anos de 2011 e 2018 para analisar as modificações ocorridas durante o período de estiagem. A Bacia Hidrográfica do açude Cachoeira II apresenta forma alongada, o que indica uma baixa tendência a enchentes em condições normais de precipitação, baixa sinuosidade ocasionando uma maior velocidade de escoamento da água até o exutório e apresenta predominância das seguintes classes de relevo: suave ondulado e ondulado. Os dados de uso e ocupação demostraram que a maior ocupação da bacia é de formação do bioma Caatinga. A estiagem no período de 2012 a 2017 junto com a diminuição das áreas de caatinga e o aumento das áreas de pastagem favoreceram a ocorrência de erosão e o transporte de sedimentos na bacia, contribuindo para diminuição do açude Cachoeira II.

Palavras-Chave: MAPBIOMAS, QGIS, SRTM

\section{Morphometric characterization and land use and occupation in a watershed in the semiarid of Pernambuco}

\begin{abstract}
This work investigated the characterization of a watershed in the Semiarid of Pernambuco and the effects of the drought on the conditions of use and occupation of the soil through the adoption of geotechnologies applied to the management of water resources. For that, the altimetry images were obtained by Embrapa and an analysis for morphometric characterization was performed with the QGIS software and the Terrain Analysis Using Digital Elevation Models - TauDEM extension. In addition, the images of land use and occupation on the MAPBIOMAS BRASIL platform for the years 2011 and 2018 were used to analyze the changes that occurred during the drought period. The Cachoeira II reservoir watershed has an elongated shape, which indicates a low tendency to flooding under normal precipitation conditions, low sinuosity causing a faster flow of water to the exutory and presents predominance of the following relief classes: smooth undulating and wavy. The use and occupation data showed that the largest occupation of the basin is the formation of the Caatinga biome. The drought in the period from 2012 to 2017 together with the decrease in the caatinga areas and the increase in the pasture areas favored the occurrence of erosion and the transport of sediments in the basin, contributing to the decrease of the Cachoeira II reservoir.
\end{abstract}

Keywords: MAPBIOMAS, QGIS, SRTM

\section{Introdução}

A água é um recurso fundamental para as atividades humanas, sua gestão demanda ações complexas em planejamento, outorga e fiscalização de concessões de uso, coordenação dos múltiplos agentes setoriais e monitoramento de sua quantidade e qualidade (Campos e Vieira, 1993). Para tanto, o conhecimento das condições

Santos. A. G. B.; Bezerra. C. A.; Nascimento. C. H. A. Souza. B. S. L.; Silva. B. L. J.; Moura. A. B. G. 
da superfície terrestre, nas forma de uso e ocupação do solo, em conjunto com as características morfométricas auxiliam na compreensão da dinâmica da água, logo que, influenciam nos processos relativos ao ciclo hidrológico (i.e. infiltração, evapotranspiração e escoamento superficial) bem como na na sua qualidade (Oliveira et al, 2018).

As regiões áridas e semiáridas recobrem grande parte da superfície continental do globo e requerem uma atenção ainda maior no que se refere aos recursos hídricos, pois apresentam alta propensão à escassez hídrica (Araújo et al, 2019). As regiões semiáridas do Nordeste do Brasil possuem uma condição climática caracterizada por altas taxas de evaporação e baixos volumes de precipitação irregulares no espaço e tempo, o que favorece a ocorrência de eventos severos de seca ao longo do tempo. A seca depende da duração, severidade, cobertura da superfície terrestre e impactos causados pelo déficit hídrico (Brito et al., 2018) e é um fenômeno natural ocasionado das condições climáticas e criam situações críticas e riscos às populações das regiões residentes dos locais afetados (Marengo et al., 2017). Para amenizar esta situação, reservatórios foram construídos com o objetivo de garantir a manutenção da disponibilidade hídrica por mais tempo e assegurar o uso múltiplo da água nestas regiões. Nesse contexto, a bacia hidrográfica do açude Cachoeira II é uma sub-bacia do Rio Pajeú no sertão Pernambucano, que sofre com a influência do clima da região, e contribui com o abastecimento de água da cidade de Serra Talhada - PE, tendo uma capacidade de 21 milhões de metros cúbicos de água na sua capacidade máxima (COMPESA, 2018), no qual a água desse reservatório favorece a população local, tanto no fornecimento de água para abastecimento público, irrigação e pesca.

As características morfometricas e de ocupação do uso do solo foram estudadas por diferentes autores (Silva et al., 2017; Lopes et al., 2018; Neta et al, 2018; Ficher et al., 2019). Na Bacia do rio Pajeú, que representa a de maior extensão espacial do estado de Pernambuco, um estudo desenvolvido por Feitosa et al. (2011) avaliaram as condições morfométricas de toda a bacia para identificar a dinâmica e fragilidades ambientais na bacia. Contudo, não há estudos da caracterização morfométrica e uso e ocupação dos reservatórios na bacia do Pajeú.

Portanto, esse estudo investigou a caracterização morfométrica de uma bacia hidrográfica do Açude Cachoeira II e os efeitos da estiagem nas condições uso e ocupação do solo com uso de geotecnologias.

\section{Material e métodos}

$O$ estudo foi realizado na bacia hidrográfica do açude Cachoeira II, localizado no município de Serra Talhada, Pernambuco - Brasil, Bacia hidrográfica do rio Pajeú. O clima da região é semiárido quente, segundo Köppen é classificado por BSh, o qual se caracteriza por chuvas escassas, irregularidade em sua distribuição e temperaturas médias em torno de 27 ${ }^{\circ} \mathrm{C}$. O volume anual de precipitação está entre 250 a $750 \mathrm{~mm}$, ocorrendo em poucos meses e de forma concentrada (Embrapa, 2019). A bacia hidrográfica do Açude Cachoeira II, localizado na latitude $7^{\circ} 58^{\prime} 7.98^{\prime \prime}$ Sul e na longitude $38^{\circ} 19^{\prime} 57.75^{\prime \prime}$ Oeste, com uma altitude de 435 metros (Figura 1), é endorréica, ou seja o escoamento não se dá de modo continuo até o Oceano (Christofoletti, 1980), convergindo neste caso, para o Rio Pajeú. 


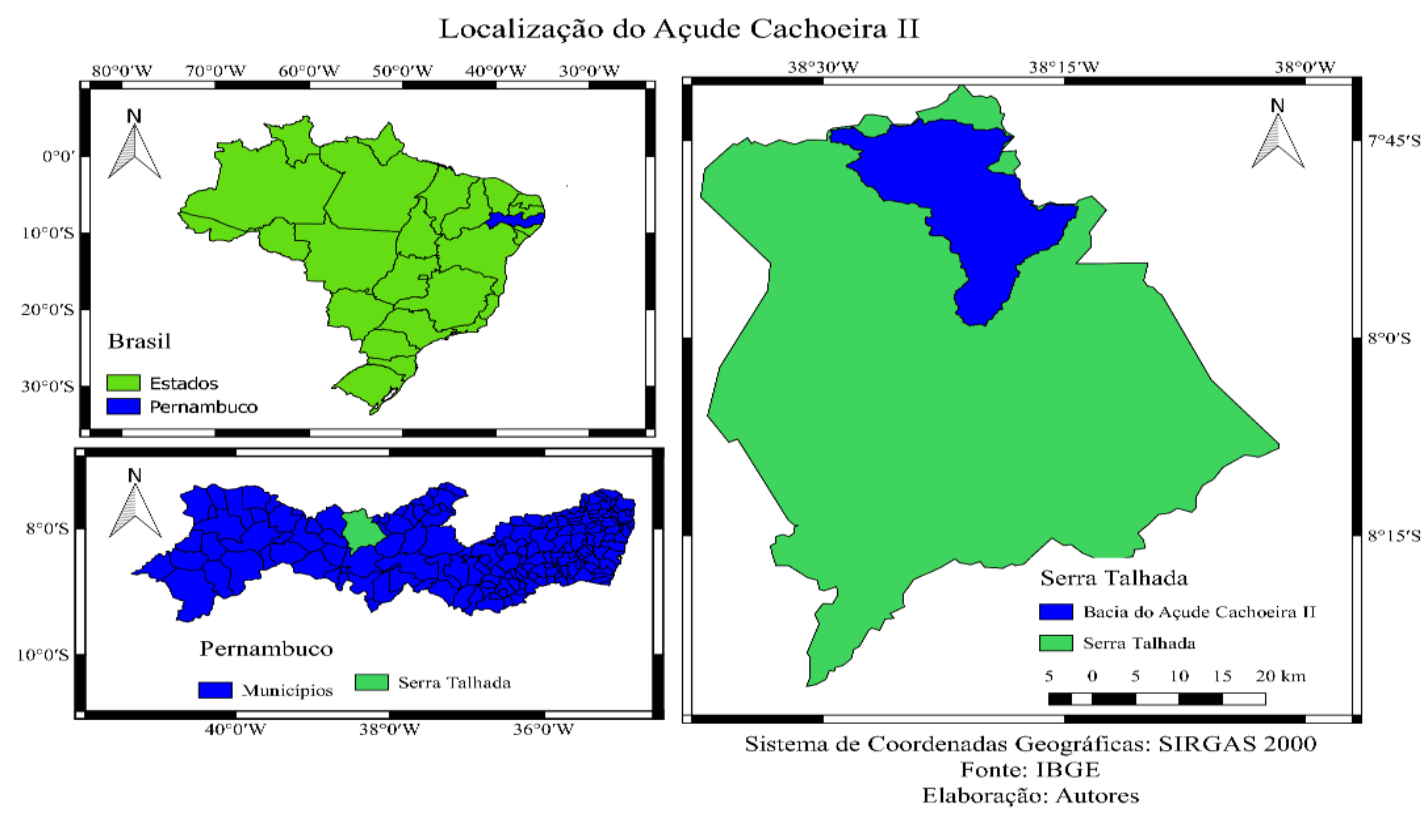

Figura 1. Localização espacial da sub-bacia hidrográfica do açude Cachoeira II.

As análises da caracterização morfométrica utilizaram dados oriundos das imagens de radar interferométricas da SRTM (Shuttle Radar Topography Mission), com resolução espacial de 30 metros, disponibilizados pelo Instituto Nacional de Pesquisas Espaciais INPE (Valeriano e Rosseti, 2012). A montagem do processamento digital da imagem foi desenvolvida no software Quantum GIS (QGIS) versão 2.18.24, com o auxilio do complemento de análises hidrológicas: Terrain Analysis Ucantar Digital Elevation Models - TauDEM com base nos Modelos Digitais de Elevação (MDEs), essa ferramenta consiste em uma biblioteca de algoritmos de análises hidrológicas, usado para os seguintes processos: Remover Depressões (Pit Remove); Direção de Fluxo (Flow Direction) pelo método D-8; Área de Contribuição (Contributing Area) pelo método D-8; Rede de Drenagem (Stream Definition by Threreshold).

A verificação dos dados processados no QGIS resultou na obtenção dos parâmetros morfométricos relativos às características geométricas, do relevo e da rede de drenagem. Sendo estes: a área total da bacia; perímetro total da bacia; coeficiente de compacidade; fator de forma; índice de conformidade; diferença entre altitude máxima, média e mínima; ordens dos canais, de acordo com o esquema de ordenação de Strahler (1952); comprimento total dos rios; comprimento do rio principal; densidade de drenagem; declividade média do rio principal; sinuosidade do rio principal; índice de sinuosidade; tempo de concentração, segundo Kirpich (1940), visto na Tabela 1.
Tabela 1. Parâmetros morfométricos e fórmulas.

\begin{tabular}{c|c}
\hline $\begin{array}{c}\text { Parâmetros } \\
\text { Morfométricos }\end{array}$ & Fórmulas \\
\hline $\begin{array}{c}\text { Coeficiente de } \\
\text { Compacidade }\end{array}$ & $\mathrm{Kc}=0,28 \frac{\mathrm{P}}{\sqrt{\mathrm{A}}}$ \\
\hline $\begin{array}{c}\text { Fator de Forma } \\
\text { Densidade de } \\
\text { drenagem }\end{array}$ & $\mathrm{Dd}=\frac{\mathrm{A}}{\mathrm{Lx}}$ \\
\hline $\begin{array}{c}\text { Índice de sinuosidade } \\
\text { Índice de sinuosidade } \\
(\%)\end{array}$ & $\mathrm{Is}=\frac{\mathrm{L}}{\mathrm{dv}}$ \\
$\begin{array}{c}\text { Tempo de } \\
\text { concentração }(\mathbf{h})\end{array}$ & $\mathrm{100}(\mathrm{L}-\mathrm{Lt})$ \\
$\mathrm{Lc}=1,42\left(\frac{\mathrm{L}^{3}}{\Delta \mathrm{H}}\right)^{0,385}$
\end{tabular}

Em que: $\mathrm{A}=$ área da bacia $\left(\mathrm{km}^{2}\right) ; \mathrm{P}=$ perímetro da bacia $(\mathrm{km}) ; \mathrm{L}=$ comprimento do canal principal $(\mathrm{km}) ; \mathrm{Lx}=$ comprimento axial da bacia $(\mathrm{km}) ; \mathrm{C}=$ comprimento total dos canais $(\mathrm{km}) ; \mathrm{dv}=$ distância vetorial entre os pontos extremos do canal principal $(\mathrm{km}) ; \mathrm{Lt}=$ distância vetorial entre os pontos extremos do canal principal $(\mathrm{km}) ; \Delta \mathrm{H}=$ desnível máximo da bacia (m).

Para compreender a dinâmica de mudança de uso e cobertura do solo na Bacia do Açude Cachoeira II e seus possíveis efeitos sobre o

Santos. A. G. B.; Bezerra. C. A.; Nascimento. C. H. A. Souza. B. S. L.; Silva. B. L. J.; Moura. A. B. G. 
reservatório de água, foram utilizadas as imagens fornecidas pelo Projeto MAPBIOMAS BRASIL dos anos de 2011 e 2018 (https://code.earthengine.google.com/?accept_rep $\mathrm{o}=$ users/mapbiomas/user-toolkit). A escolha desses anos foi devido ao interesse de observar como a bacia se comportava com os efeitos da estiagem, dos anos 2012 até 2016, conforme Lins et al. (2017). A Figura 2 apresenta os dados de precipitação de Serra Talhada obtidos na Agência Pernambucana de Águas e Clima (APAC) os quais evidenciam que após o período seco, os anos de 2017 e 2018 foram próximos aos valores médios para o local, que para o período de 1987 até 2019 correspondeu à $675,5 \mathrm{~mm}$ ao ano.

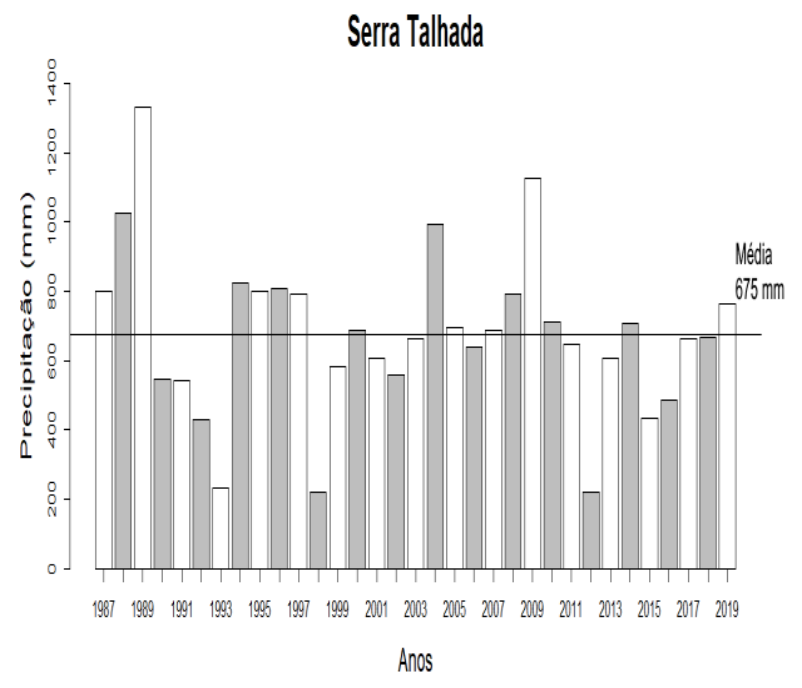

Figura 2. Dados de precipitação de Serra Talhada entre o período de 1987 até 2019.

As imagens obtidas no MAPBIOMAS BRASIL foram reprojetadas para o datum SIRGAS2000 e, em seguida, recortadas com o arquivo vetorial da bacia do Açude Cachoeira II, obtida nas etapas anteriores. Posteriormente, foi realizada uma contagem da área de cada classe com uso da r.report. Com isso, foi possível perceber as mudanças ocorridas ao longo do período estudado. Por fim, houve a confecção de mapas temáticos com os dados da altimetria, declividade, rede de drenagem e uso e ocupação do solo, que permitiram compreender a variabilidade espacial dessas informações.

\section{Resultados e discussão}

Os índices morfométricos e medidas geométricas da Bacia Hidrográfica do açude Cachoeira II encontram-se na Tabela 2. As medidas da bacia hidrográfica do açude Cachoeira II mostram uma área de $347,6724 \mathrm{~km}^{2}$ e perímetro de 147,6143 km. Os parâmetros das características geométricas Fator de Forma $(\mathrm{Kf})=0,3646$ e Coeficiente de Compacidade $(\mathrm{Kc})=2,2167 \mathrm{da}$ bacia do açude Cachoeira II indicam uma baixa tendência a enchentes em condições normais de precipitação de acordo com Villela e Mattos (1975) (Tabela 2). De acordo com estes autores valores de Kc próximos à unidade indicam maior propensão à ocorrência de enchentes.

A interpretação combinada destes parâmetros indica que a bacia apresenta uma forma comprida e ambientalmente com tendências a conservação da ocupação do solo, segundo a classificação ambiental proposto por Nardini et al. (2013). Os dados obtidos demostram que em condições climatológicas normais a bacia do açude Cachoeira II não apresenta tendência de ocorrer inundações no seu exutório.

De acordo com a densidade de drenagem encontrada de $0,7644 \mathrm{~km} \mathrm{~km}^{-2}$, a bacia possui uma capacidade de drenagem regular (Villela et al, 1975), e é classificada ambientalmente com baixo escoamento superficial e maior infiltração (Nardini et al., 2013), demostrando a fragilidade da bacia em drenar água até o seu exutório. Tais constatações podem ser decorrentes da associação de fatores climáticos (i.e. baixas pluviosidades e elevadas taxas de evapotranspiração) e geomorfológicas (baixas declividades) o que interfere no escoamento global da bacia. Resultados semelhantes foram observados por Feitosa et al. (2011) para a Bacia Hidrográfica do rio Pajeú.

A sinuosidade do curso d'água de 1,3556 $\mathrm{km} \mathrm{km}^{-1}$, indica situação intermediária entre formas regulares e irregulares (Freitas, 1952). O índice de sinuosidade de 26,2339\% indica que o canal principal tem tendência reta (Mansikkaniemi, 1970). Analisando estes valores em conjunto, segundo Santos et al. (2012), quanto maior a sinuosidade, maior será a dificuldade de se atingir o exutório do canal, portanto, a velocidade de escoamento será menor o que influência no maior tempo de escoamento até o seu exutório.

Para estes valores destaca-se que a bacia do Cachoeira II por apresentar baixa sinuosidade tem uma maior velocidade de escoamento, como pode ser observado também tempo de concentração de 5,23 horas (Kirpich). A rede de drenagem da bacia hidrográfica do açude Cachoeira II foi considerada de $4^{\text {a }}$ ordem (Strahler, 1952) como pode ser observado na Figura 3. 
Tabela 2. Medidas e valores dos parâmetros morfométricos da bacia do açude Cachoeira II, Serra Talhada-PE.

\begin{tabular}{|c|c|}
\hline Parâmetros & Resultados \\
\hline \multicolumn{2}{|c|}{ Características geométricas } \\
\hline Área total $\left(\mathrm{A}, \mathrm{km}^{2}\right)$ : & 347,6724 \\
\hline Perímetro total $(\mathrm{P}, \mathrm{km})$ : & 147,6143 \\
\hline $\begin{array}{c}\text { Coeficiente de Compacidade } \\
\text { (Kc): }\end{array}$ & 2,2167 \\
\hline Fator de Forma (Kf): & 0,3646 \\
\hline \multicolumn{2}{|c|}{ Características de relevo } \\
\hline Altitude máxima (m): & 848 \\
\hline Altitude média (m): & 562,223 \\
\hline Altitude mínima (m): & 440 \\
\hline \multicolumn{2}{|c|}{ Caracterização da rede de drenagem } \\
\hline Ordem: & 4 \\
\hline $\begin{array}{l}\text { Comprimento total dos rios } \\
\qquad(\mathrm{km}) \text { : }\end{array}$ & 265,749 \\
\hline $\begin{array}{l}\text { Comprimento do rio } \\
\text { principal }(\mathrm{km}) \text { : }\end{array}$ & 22,936 \\
\hline $\begin{array}{l}\text { Densidade de drenagem } \\
\qquad\left(\mathrm{km} / \mathrm{km}^{2}\right):\end{array}$ & 0,7644 \\
\hline $\begin{array}{l}\text { Declividade média do rio } \\
\text { principal }(\%) \text { : }\end{array}$ & 1,7789 \\
\hline $\begin{array}{l}\text { Sinuosidade do rio principal } \\
\qquad(\mathrm{km} / \mathrm{km}):\end{array}$ & 1,3556 \\
\hline Índice de sinuosidade (\%): & 26,2339 \\
\hline Tempo de concentração (h): & 5,2309 \\
\hline
\end{tabular}

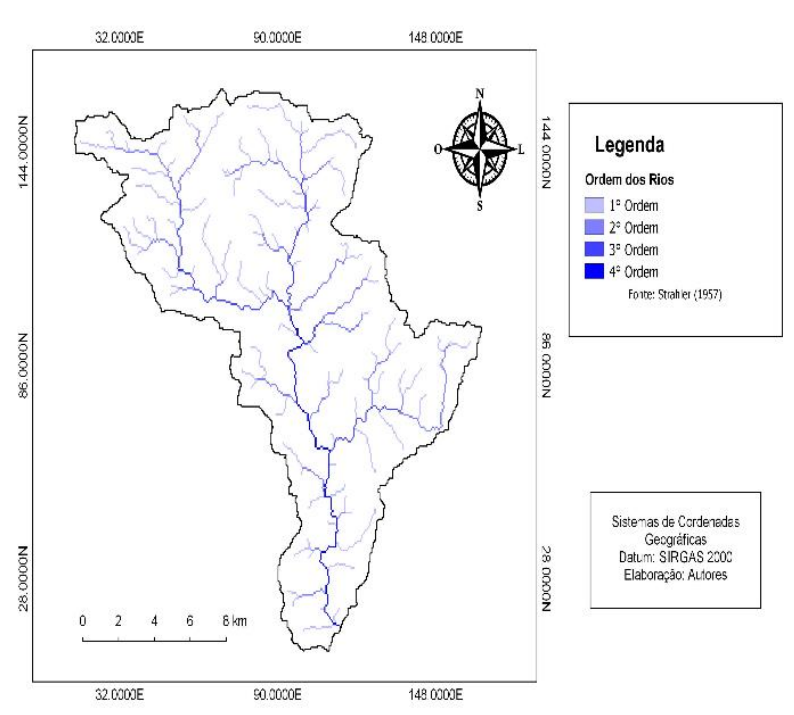

Figura 3. Ordenamento da Rede de Drenagem do açude Cachoeira II pela classificação de Strahler (1952).

Analisando a declividade do terreno da bacia (Figura 4), verifica-se predominância das Santos. A. G. B.; Bezerra. C. A.; Nascimento. C. H. A. Souza. B. S. L.; Silva. B. L. J.; Moura. A. B. G. classes de relevo ondulado e suavemente ondulado (Embrapa, 1979), com valores de $13.995,70$ ha e 12.426,58 ha, respectivamente. Para as classes de relevo forte-ondulado, plano e montanhoso as áreas totais são de 5.475,41 ha, $1.205,31$ ha e 1.097,25 ha, respectivamente. As menores áreas de relevo pertencem à classe fortemontanhoso com 68,22 ha. Regiões que apresentam declividade suave ondulada podem ser desenvolvidas atividades como a agricultura e a pecuária utilizando-se práticas simples de manejo do solo (Mioto et al., 2017).

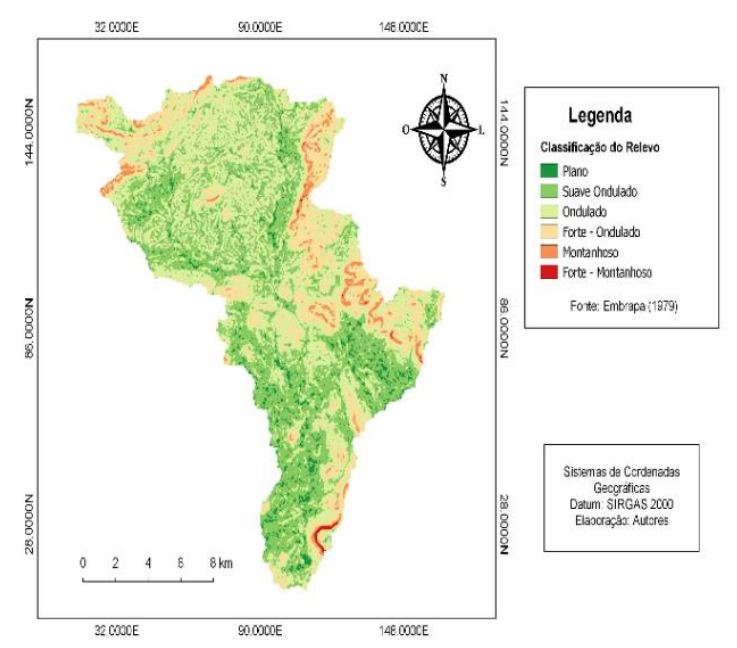

Figura 4. Classificação do relevo da bacia do açude Cachoeira II.

Em relação aos principais uso e ocupação do solo da bacia do açude Cachoeira II no ano de 2011 (Figura 5), antes do período de estiagem, e 2018, após a estiagem, observa-se a predominância da formação do bioma de Caatinga (MAPBIOMAS BRASIL, 2019), com 21.688,35 ha e 21.174,66 ha, respectivamente. Além disso, é possível observar um aumento das áreas de pastagem e campestre, com pixel de tons mais amarelo e uma nítida redução da área de água do açude Cachoeira II. A tabela 3 apresenta as áreas e os diferentes tipos de uso e ocupação do solo.

Analisando a Tabela 3, observa-se que a área de pastagem aumentou entre os anos de 2011 e 2018, em torno de 639,64 ha. Enquanto a formação florestal ocorreu um aumento de 183,81 ha, entre 2011 e 2018, enquanto a área de Caatinga houve uma redução de 512,83 ha. O mosaico de agricultura e pastagem teve um redução entre 2011 e 2018, na ordem de 249,68 ha, e diminuição de 76,18 ha nas áreas do açude Cachoeira II, devido à estiagem. 


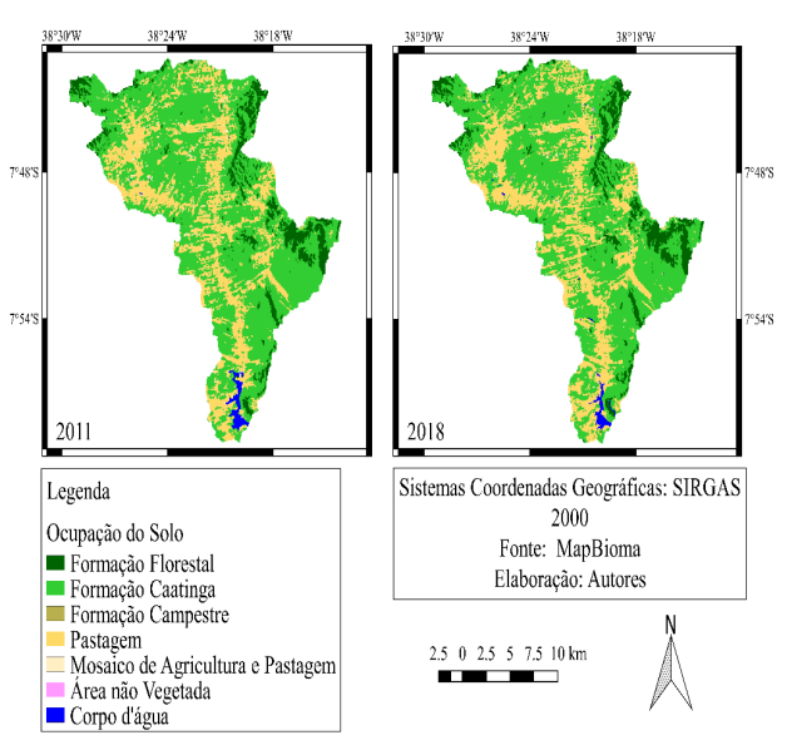

Figura 5. Uso e ocupação do solo na bacia hidrográfica do açude Cachoeira II nos anos de 2011 e 2018.

Tabela 3. Quantificação das áreas de uso e ocupação do solo nos anos de 2011 e 2018.

\begin{tabular}{ccc}
\hline Tipos de Uso e & $\mathbf{2 0 1 1}$ & $\mathbf{2 0 1 8}$ \\
\cline { 2 - 3 } Ocupação do Solo & \multicolumn{2}{c}{ Área (ha) } \\
\hline Formação Florestal & $2.892,31$ & $3.075,29$ \\
Formação Caatinga & $21.768,86$ & $21.255,20$ \\
Formação Campestre & 99,56 & 104,90 \\
Pastagem & $8.884,93$ & $9.523,64$ \\
Mosaico de & & \\
Agricultura e & 629,77 & \\
Pastagem & & 379,27 \\
Área não Vegetada & 83,43 & 97,56 \\
Água & 402,34 & 325,34 \\
Total & $\mathbf{3 4 . 7 6 1 , 2 0}$ & $\mathbf{3 4 . 7 6 1 , 2 0}$ \\
\hline
\end{tabular}

No geral os efeitos das mudanças na ocupação do solo sobre o reservatório de água ocorreram por conta do período de estiagem que influenciou na diminuição da área do açude Cachoeira II e com aumento das áreas não vegetadas e pastagem e diminuição da área de Caatinga, caraterísticas que favorecem o aumento da vulnerabilidade da bacia hidrográfica à erosão hídrica (Bruno, 2017).

Mesmo com a maior parte da bacia sendo ocupada por formação de Caatinga, é importante observar que as matas ciliares dos cursos não estão sendo observadas. Inclusive, as atividades agropastoris se concentram às margens dos cursos d'águas na bacia em questão e, em geral, as propriedades adotam práticas inadequadas de produção muitas vezes devido à falta de conhecimento técnico (Morais, 2019).
Essas condições associada às características morformétricas, de maior propensão de velocidade de escoamento, potencializam os processos erosivos e transporte de materiais orgânicos e inorgânicos para os cursos d'água. Como consequência ocorre o assoreamento do açude e, dessa forma, uma redução do volume útil do reservatório (Ferreira et al., 2019).

Uma solução para contribuir para a preservação da bacia e das condições de produção de água seria a adoção de pagamentos de serviços ambientais para os produtores da região, que é um mecanismo de gerenciamento dos ecossistemas, valoração do serviços ecossistêmicos e incentivo ao agentes locais (Souza et al., 2018). Outro ponto que pode auxiliar na melhoria da condição da bacia é a extensão rural que pode ser promovida por órgãos federais, estaduais e municipais. Uma vez que a capacitação das comunidades para práticas que busquem pela conservação e manejo sustentável é uma necessidade fundamental para atingir a sustentantibilidade (Kaufmann et al, 2016).

\section{Conclusões}

A Bacia Hidrográfica do açude Cachoeira II indica uma baixa tendência a enchentes em condições normais de precipitação.

As classes de relevo predominantes na bacia hidrográfica são suave-ondulado e ondulado.

Os dados de uso e ocupação do solo demostrou que a bacia possui maior predominância de formação vegetal da Caatinga.

A estiagem no período de 2012 a 2017 junto com a diminuição das áreas de caatinga e o aumento das áreas de pastagem influenciaram na redução da disponibilidade hídrica do açude Cachoeira II.

\section{Agradecimentos}

Os autores agradecem à Universidade Federal Rural de Pernambuco pelo apoio e infraestrutura à pesquisa, à EMBRAPA pela disponibilização das imagens de altimetria e ao Projeto MAPBIOMAS BRASIL pela disponibilização das imagens de uso e ocupação do solo.

\section{Referências}

Araújo, M. D., Ribeiro, M. M. R., Braga, C. F. C., 2019. Integrando a modelagem da alocação de

Santos. A. G. B.; Bezerra. C. A.; Nascimento. C. H. A. Souza. B. S. L.; Silva. B. L. J.; Moura. A. B. G. 
água ao sistema de indicadores FPEIR: aplicação ao semiárido do Brasil. Engenharia Sanitaria e Ambiental 24, 6, 1167-1181.

Brito, S. S. B., Cunha, A. P. M., Cunningham, C. C., Alvalá, R. C., Marengo, J. A., Carvalho, M. A, 2018. Frequency, duration and severity of drought in the Semiarid Northeast Brazil region. International Journal of Climatology $38,2,517-529$.

Bruno, L.O., 2017. GRASS: A free and open source solution for hydrographic body analysis. Nativa 5, 1, 24-30.

Campos, J. N. B., Vieira, V. P. P. B., 1993. Gerenciamento de recursos hídricos: a problemática do Nordeste. Administração Pública . 27, 81-91.

Christofoletti, A., 1980. Geomorfologia, $2^{\text {a }}$ edição. Editora Edgard Blucher, São Paulo 188 p.

COMPESA, Companhia Pernambucana de Saneamento, 2018. Barragem Cachoeira II se recupera e melhora abastecimento de Serra Talhada Disponível em: $<$ https://servicos.compesa.com.br/barragemcachoeira-ii-se-recupera-e-melhoraabastecimento-de-serra-talhada/>. Acesso em: 02 dez. 2019.

EMBRAPA, Empresa Brasileira de Pesquisa Agropecuária, 2019. Disponível em: <https://www.cnpf.embrapa.br/pesquisa/efb/cli ma.htm>. Acesso em: 08 dez. 2019.

EMBRAPA, Empresa Brasileira de Pesquisa Agropecuária - Serviço Nacional de Levantamento e Conservação de Solos (Rio de Janeiro, RJ). In: reunião técnica de levantamento de solos, 10.1979, Rio de Janeiro. Súmula... Rio de Janeiro, 1979. 83p. (EMBRAPA-SNLCS. Miscelânea, 1).

Feitosa, A., Santos, B., Araujo, M. S. B., 2011. Caracterização Morfométrica e identificação de Áreas Susceptíveis a Erosão na Bacia do Rio Pajeú, PE: o Estudo de Caso da Bacia do Rio Pajeú/PE. Revista Brasileira de Geografia Física, 3, 820-836.

Ferreira, N. C. F., Mello Duarte, J. R., Oliveira, L. A. B., Silva, E. C., Carvalho, I. A., 2019. O papel das matas ciliares na conservação do solo e água. Biodiversidade 18, 3, 171-179.

Ficher, K. N., Pereira, D. R., Oliveira, J. S., Almeida, A. Q., Uliana, E. M., 2019. Assessment of digital elevation models to obtain morphometric characteristics in relief transition region. Revista Ambiente \& Água 14, 1, e2280.
Freitas, R. O., 1952. Textura de drenagem e sua aplicação geomorfológica. Boletim Paulista de Geografia 11, 53-57.

Kaufmann, M. P.; Reiniger, L. R. S.; Wizniewsky, J. G.; Muniz, M. F. B., 2016. Resgate e conservação da agrobiodiversidade crioula em Ibarama-RS: estratégias de manutenção. Extensão Rural 23, 4, 66-78.

Kirpich, Z. P., 1940. Time of concentration of small agricultural watersheds. Civil Engineering, Reston 10, 6, 362.

Lins, F. A. C., Silva, J. L. B., Moura, G. B. A., Ortiz, P. F. S., Oliveira, J. D. A., Alves, M. V. C., 2017. Quantile technique to precipitation, rainfall anomaly index and biophysical parameters by remote sensing in Serra Talhada, Pernambuco. Journal of Hyperspectral Remote Sensing 7, 6, 334-344.

Lopes, I., Leal, B. G., Ramos, C. M. C., 2018. Morphometric characterization of the basin in the semi-arid region of Pernambuco using SRTM data in free software. Journal of Hyperspectral Remote Sensing 8, 1, 31-40.

Mansikkaniemi, H., 1970. The sinuosity of rivers in northern Finland. Publicationes Instituti Geographici Universitatis Turkuensis 52, 1632.

Marengo, J. A., Torres, R. R., Alves, L. M., 2017. Drought in Northeast Brazil-past, present, and future. Theoretical and Applied Climatology 129, 3-4, 1189-1200.

Mioto, C. L., Ribeiro, V. O., Souza, D. M. Q., Pereira, T. V., Anache, J. A. A., Paranhos Filho, A. C., 2017. Morfometria de bacias hidrográficas através de SIGs livres e gratuitos. Anuário do Instituto de Geociências 37, 2, 1622.

Morais, J. R. G., 2019. Estabelecimentos Rurais Camponeses no Bioma Caatinga de clima semiárido: perspectivas e desafios na atualidade. Revista Brasileira de Meio Ambiente, 7, 1, 29-47.

Nardini, R. C., Pollo, R. A., Barros, Z. X., Cardoso, L. G., Gomes, L. N., 2013. Análise morfométrica e simulação das Áreas de Preservação Permanente de uma microbacia hidrográfica. Irriga 18, 4, 687-699.

Neta, S. R. A., Bias, E. S., Santos, C. A. M., 2018. Classificação Baseada em Objetos e Mineração de Dados: Aplicação de Ferramentas Open Source no Mapeamento de Área Urbana. Revista Brasileira de Cartografia, v. 70, n. 3, p. 932-966, 2018.

Oliveira, D. H. R., Acorsi, M. G., Smaniotto, D. A., 2018. Uso e ocupação do solo e caracterização morfométrica de microbacia na 
região centro-sul paranaense. Águas Subterrâneas 32, 2, 1-10.

Projeto MapBiomas - Coleção 2019 da Série Anual de Mapas de Cobertura e Uso de Solo do Brasil, acessado em 02 de Dezembro de 2019 através de https://code.earthengine.google.com/?accept_r epo=users/mapbiomas/user-toolkit $>$.

Santos, A. M., Targa, M. S., Batista, G. T., Dias, N. W., 2012. Análise morfométrica das subbacias hidrográficas Perdizes e Fojo no município de Campos de jordão, SP, Brasil. Revista Ambiente \& Água 7, 3, 195-211.

Silva, C. V. T., Ribeiro Filho, J. C., Silva, P. C. M., Aquino Lemos Filho, L. C., Brasil, J. B., 2017. Caracterização Morfométrica, Uso E Ocupação De Uma Bacia Hidrográfica. Revista Engenharia na Agricultura 25, 5, 436-444.
Souza, V. V. C., Gallardo, A. L. C. F., Côrtes, P. L., Fracalanza, A., Ruiz, M. S., 2018. Pagamento por serviços ambientais de recursos hídricos em áreas urbanas: perspectivas potenciais a partir de um programa de recuperação da qualidade de água na cidade de São Paulo. Cadernos Metrópole 20, 42, 493512.

Strahler, A. N., 1952. Hypsometric (area-altitude) - analysis of erosion al topography. Geological Society of America Bulletin 63, 10, 11171142.

Valeriano, M. M., Rossetti, D. F., 2012. Topodata: Brazilian full coverage refinement of SRTM data. Applied Geography 2, 2, 300-309.

Villela, S.M., Mattos, A., 1975. Hidrologia aplicada. São Paulo: McGraw-Hill do Brasil. $245 \mathrm{p}$. 